\title{
Cartes et légendes. Jeux sur les codes cartographiques anciens et contemporains
}

Anne-Christine Bronner, Laurent Jégou et Christine Zanin

\section{OpenEdition}

\section{Journals}

Édition électronique

URL : http://journals.openedition.org/mappemonde/799

DOI : $10.4000 /$ mappemonde.799

ISSN : 1769-7298

\section{Éditeur}

UMR ESPACE

\section{Référence électronique}

Anne-Christine Bronner, Laurent Jégou et Christine Zanin, « Cartes et légendes. Jeux sur les codes cartographiques anciens et contemporains », Mappemonde [En ligne], 125 | 2019, mis en ligne le 01 janvier 2019, consulté le 15 septembre 2020. URL : http://journals.openedition.org/mappemonde/799

Ce document a été généré automatiquement le 15 septembre 2020.

\section{(c) (i) ()




\title{
Cartes et légendes. Jeux sur les codes cartographiques anciens et contemporains
}

\author{
Anne-Christine Bronner, Laurent Jégou et Christine Zanin
}

1 Les organisateurs du colloque Temps Art \& Cartographie - La sémiologie dans tous les sens ${ }^{1}$ ont choisi d'associer à l'événement scientifique une exposition centrée sur des travaux de groupes d'étudiants tout en accueillant des œuvres soumises lors de l'appel à participation. Parallèlement, la cartothèque de la Faculté de Géographie et d'Aménagement de Strasbourg souhaitait contribuer à la manifestation en proposant des cartes anciennes issues de son fonds (Bibliothèque numérique patrimoniale du Service commun de la Documentation de l'Université de Strasbourg. Visiter la collection de la Cartothèque : http://docnum.unistra.fr/cdm/search/collection/coll18).

2 Afin d'impliquer la cartothèque à partir des cartes anciennes tout en exploitant le thème du colloque, Anne-Christine Bronner a imaginé une expérimentation cartographique s'apparentant à un jeu autour des langages (carto)graphiques, et ainsi créer un focus sur les aspects sémiologiques des cartes datant de différentes époques. Cette idée originale a été envoyée par courriel à des collègues enseignants-chercheurs et a été formulée ainsi :

3 «Je vous fais parvenir une série de cartes anciennes qui pourraient se prêter à un exercice de sémiologie, dont le résultat (la carte produite) pourrait être valorisé lors de l'exposition du colloque La sémiologie dans tous les sens. L'idée est de décrypter la sémiologie, les codes, la mise en page ou mise en scène, les attributs graphiques, la légende, les symboles, etc. de la "vieille" carte et, à partir de cette analyse, de proposer plusieurs pistes pour un exercice cartographique :

- utiliser les "codes" anciens pour représenter un espace contemporain ;

- utiliser les codes contemporains, c'est-à-dire décrypter la sémiologie, les symboles, la mise en page... embarqués dans un outil (Philcarto, QGIS, Rcarto, arcMap...), sur des interfaces en ligne (OpenStreetMap, Mappy, Michelin, GoogleMap, etc.), dans un Atlas ou autre 
publication de référence... et représenter la "vieille" carte avec une charte graphique contemporaine ».

4 Trois groupes d'étudiants (masters géomatique SIGMA et Carthagéo à Toulouse et Paris et InSituLAB, diplôme supérieur des arts appliqués à Strasbourg) ainsi que deux étudiants en licence de la Faculté de géographie et d'aménagement de Strasbourg ont travaillé sur une consigne déclinée de cette proposition de base. Les cartes, réunies sous le titre Re-cartes, ont été exposées pendant 10 jours (14-23mars 2016) au sein de l'exposition Cartologies (figure 1).

Figure 1. Le projet Re-cartes dans l'exposition Cartologies

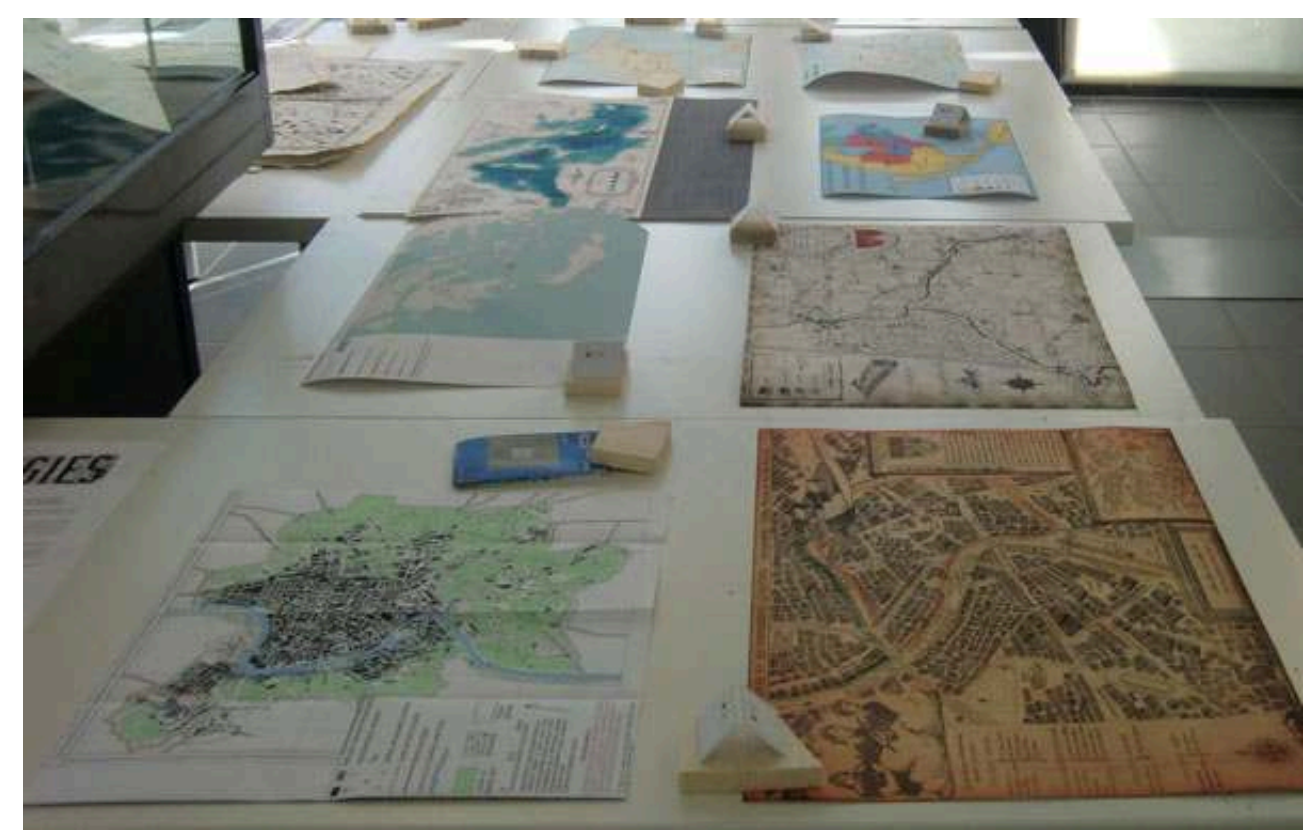

Strasbourg, 13-24 mars 2016

Photo : A.-C. Bronner, 2016

\section{Les territoires des $\mathrm{XVII}{ }^{\mathrm{e}}$ et $\mathrm{XVIII}$ siècles redessinés à l'aune des codes contemporains}

Quinze étudiants de la promotion 2015-2016 du Master Carthagéo (http:// www.pantheonsorbonne.fr/diplomes/master-professionnel-carthageo/) et Geoprisme (http://odf.univ-paris-diderot.fr/fr/offre-de-formation/master-lmd-XB/scienceshumaines-et-sociales-SHS/master-geographie-amenagement-environnement-etdeveloppement-m2-parcours-geoprisme-analyse-spaciale-et-modelisationdynamiques-spaciales-program-master-geographie-et-sciences-desterritoires-3-6.html) de Paris ont travaillé sur la consigne proposée par Christine Zanin, consigne qui divisait l'exercice en deux temps.

1. Par groupe, mener une analyse minutieuse de la carte choisie et des principes cartographiques utilisés: répertorier tous les éléments graphiques et typographiques, réfléchir à l'emprise représentée, essayer d'identifier et de contextualiser la carte dans son époque (afin de connaître son objectif et son public potentiel) ; en faire une présentation orale (diaporama). 
2. Individuellement, réaliser la même carte à l'aide d'une sémiologie et d'un style contemporain, avec la même emprise, la même projection que la carte originale.

6 L'analyse minutieuse de la sémiologie des cartes anciennes a fait suite à une recherche sur l'origine des cartes présentées: leur contexte de production et d'impression, l'objectif de la carte et son auteur, avec un gros travail de bibliographie à la clé. Il n'a pas toujours été possible de retracer cette origine, mais le contexte et l'aspect graphique des cartes ont néanmoins permis de comprendre l'usage attribué à ces cartes. À titre d'exemple, ce recensement des différents figurés pour représenter les cités et autres villages ou encore les différents modes de représentation en lien avec l'implantation géométrique (point, ligne, surface) (figure 2). Cette première phase, riche en renseignements, a permis aux étudiants de confronter les sémiologies et différentes propositions de transcriptions contemporaines pour préparer la deuxième partie de l'exercice.

Figure 2. Exemples de décryptage des cartes anciennes

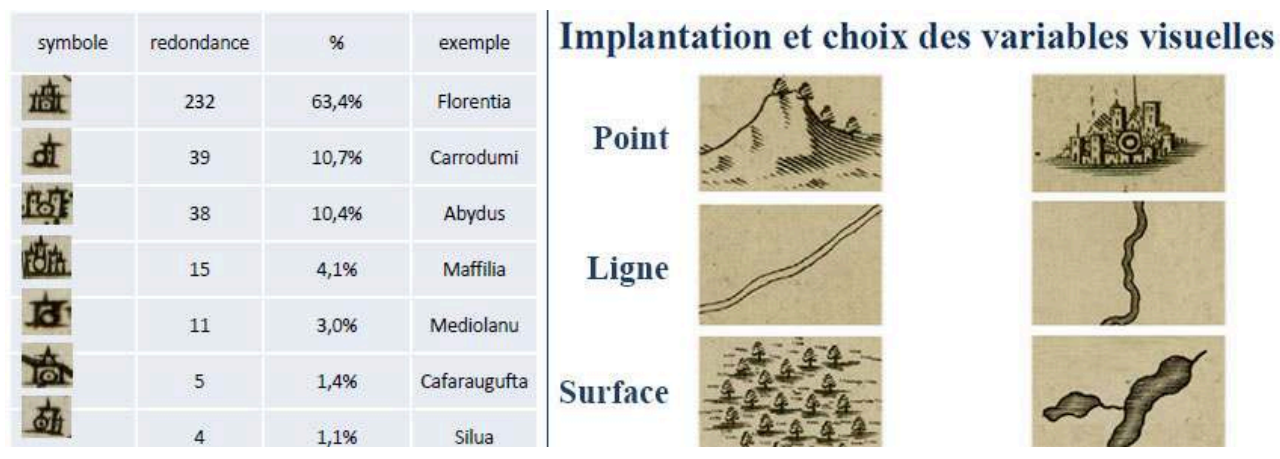

7 À partir de ces informations, de nouvelles chartes graphiques ont été mises en place par les étudiants. Deux principes ont été conservés : une charte en lien d'une part avec les principes des cartes topographiques ou de localisation (de type IGN ou Michelin), et d'autre part avec une approche plus thématique (style plan urbain ou carte mondiale). Les codes sémiologiques contemporains (basés sur la sémiologie graphique de Jacques Bertin, 1967) ont ainsi été appliqués aux informations présentées sur ces cartes. La question sémiologique a été doublée d'une question géographique : si nous utilisions des codes contemporains, fallait-il également actualiser les connaissances géographiques des lieux ? Nous avons décidé que nous allions conserver l'aspect ancien des cartes.

8 Les formes géographiques anciennes des lieux ont donc été conservées, malgré tout, les cartes ont pris bien d'autres couleurs, comme la carte (figure 3) de Hugo Egido-Sierra dont on n'excuse pas la coquille dans le titre, Provinces-unis des Pays-Bas et ses colonies, créée sur la base de la représentation Belgii pars septentrionalis communi nomine vulgo Hollandia nuncupata Continens statum potentissimce Batavorum Reipublicee, seu Provincias VII Fœderatas, cum omnium provinciarum insignibus, additis omnibus nominibus urbium et pagorum ( http://docnum.unistra.fr/cdm/compoundobject/collection/coll18/id/20/ rec/9) de Pieter Schenk éditée vers 1705, ou encore cette simulation de proposition de carte interactive de Lise Obut, au titre à améliorer, Retrouvez-vous avec Rome en cartes (figure 4) sur la base du plan de Rome Urbis Romce veteris ac Modernce Accurata Delineati (http://docnum.u-strasbg.fr/cdm/compoundobject/collection/coll18/id/8/rec/3, 
Auteur : Homann, Johann Baptist. Date : entre 1710 et 1720), source cartographique qui a inspiré plusieurs autres travaux.

9 L'ensemble des cartes a été réalisé sur le logiciel de dessin Adobe Illustrator afin de garantir les formes et la qualité graphique des documents d'origine.

Figure 3. Provinces-unis des Pays-Bas et ses colonies

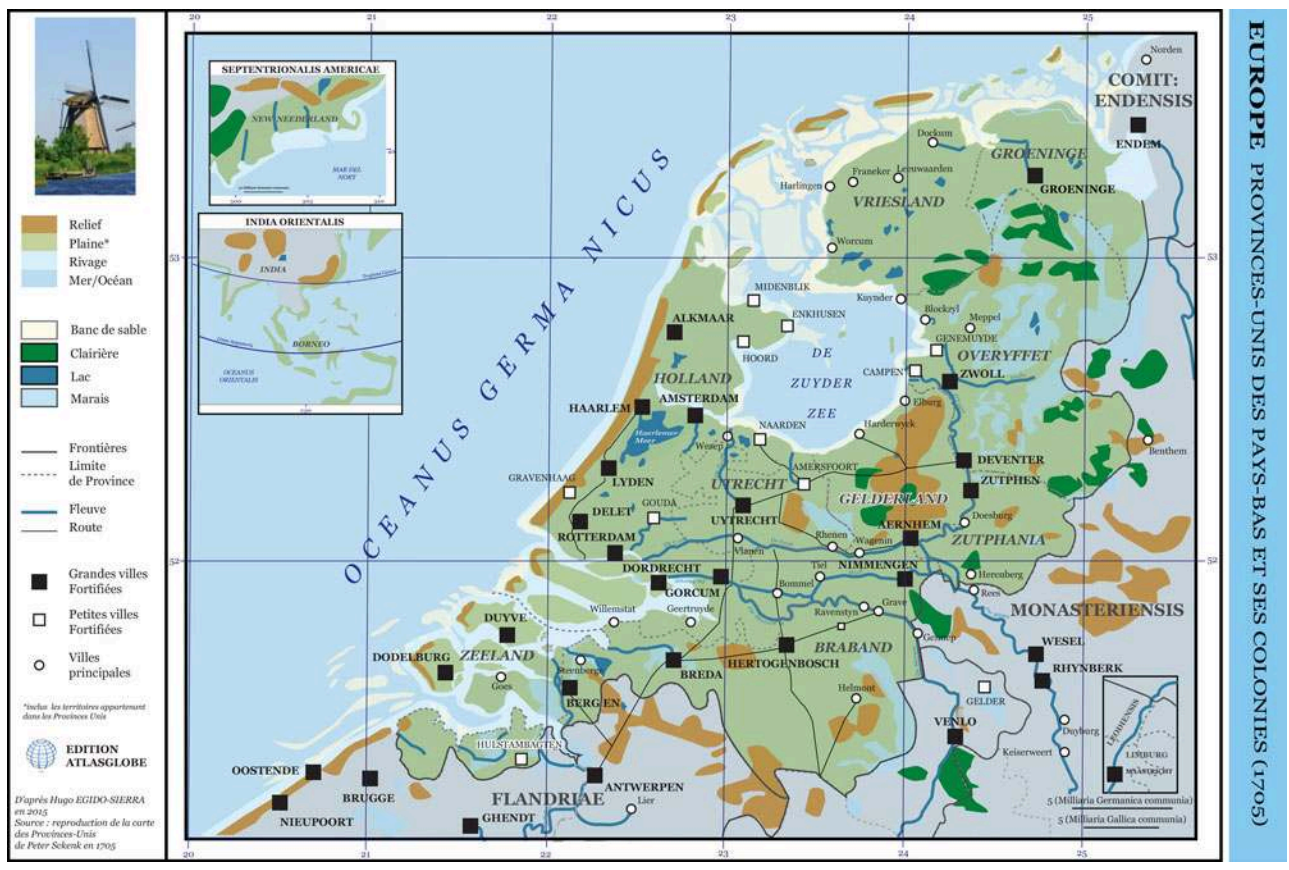

Projet Re-Cartes, exposition Cartologies, Strasbourg, 13-24 mars 2016.

Auteur : Hugo Egido-Sierra. Master Carthagéo, janvier 2016 
Figure 4. Retrouvez-vous avec Rome en cartes

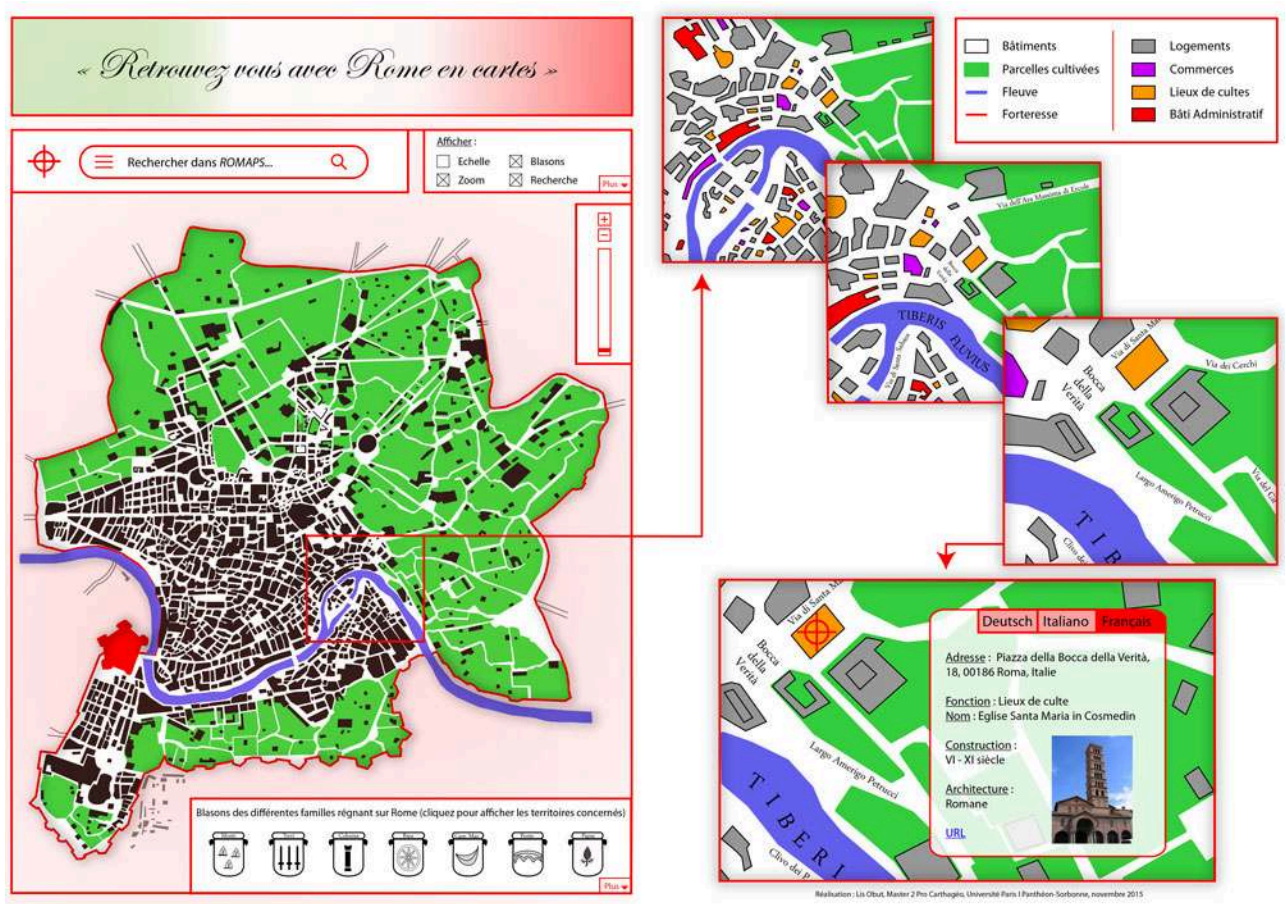

Projet Re-Cartes, exposition Cartologies, Strasbourg, 13-24 mars 2016.

Auteur : Lise Obut. Master Carthagéo, janvier 2016

Plusieurs étudiants ont choisi de jouer avec les codes des séries de cartes que l'on plie pour les glisser dans le vide-poche de la voiture, son sac ou sa poche. À titre d'exemple, les propositions de Benoît Fournier (figure 5) ou Marion Leblois (figure 6) reprennent la Carte Particulière des Pays qui sont situéz entre le Rhein, la Sarre, la Moselle et la Basse Alsace, contenant Partie du Palatinat, des Eslectorats de Mayence, et de Treves, des Eveschés de Spire et de Wormes, avec les Duchés de Deuxponts, et de Simmeren, Les Comtés de Sarbrick, Ottweiller, Bitche, Saverden, Falkenstein, Linanges, Veldents, Haut et Bas Sponheim, Les Seigneuries de Bliscastel, Landstoul, Crombach, Reypolkirch, Grevenstein, Kirn, Oberstein, Lixheim, Lautreck, Dagstoul, La Petitepierre et Dimering (http://docnum.u-strasbg.fr/cdm/ compoundobject/collection/coll18/id/26/rec/1), éditée par Hubert Jaillot vers 1705. 
Figure 5. Allemagne. Vallée de la Sarre. Situation de 1907

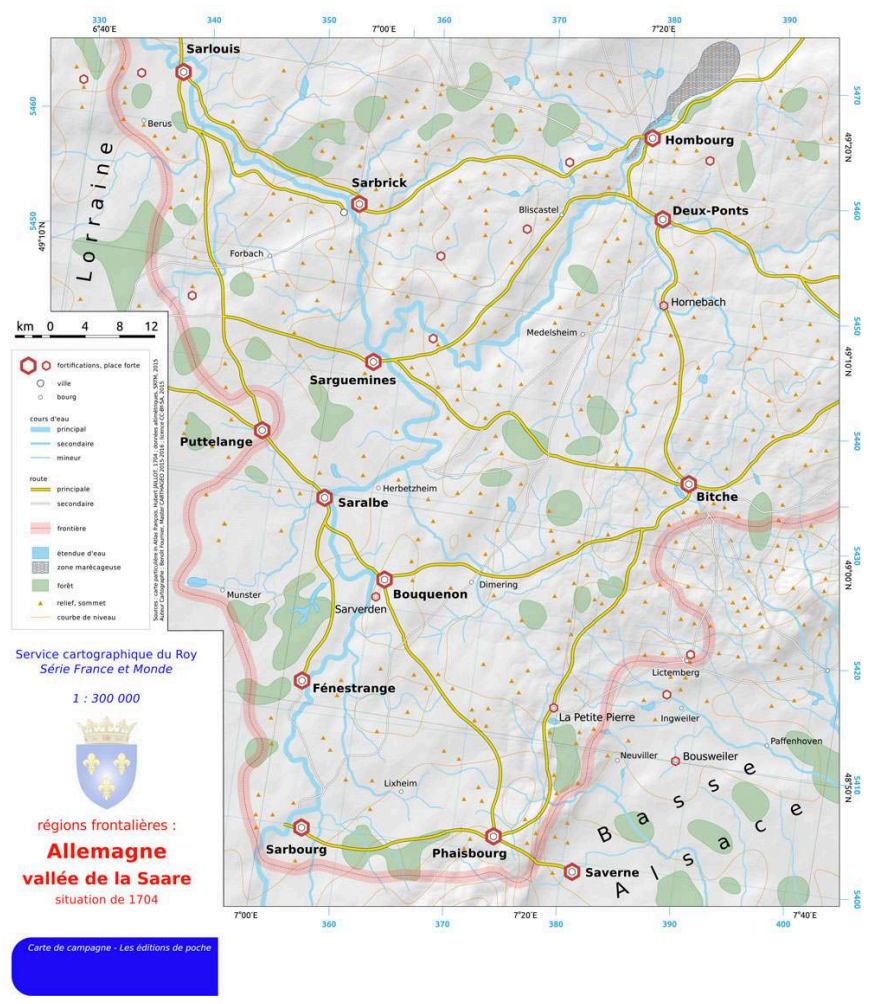

Projet Re-Cartes, exposition Cartologies, Strasbourg, 13-24 mars 2016. Auteur : Benoît Fournier. Master Carthagéo, janvier 2016

Figure 6. Carte particulière des pays qui sont situés entre le Rhin, la Sarre, la Moselle et la Basse Alsace

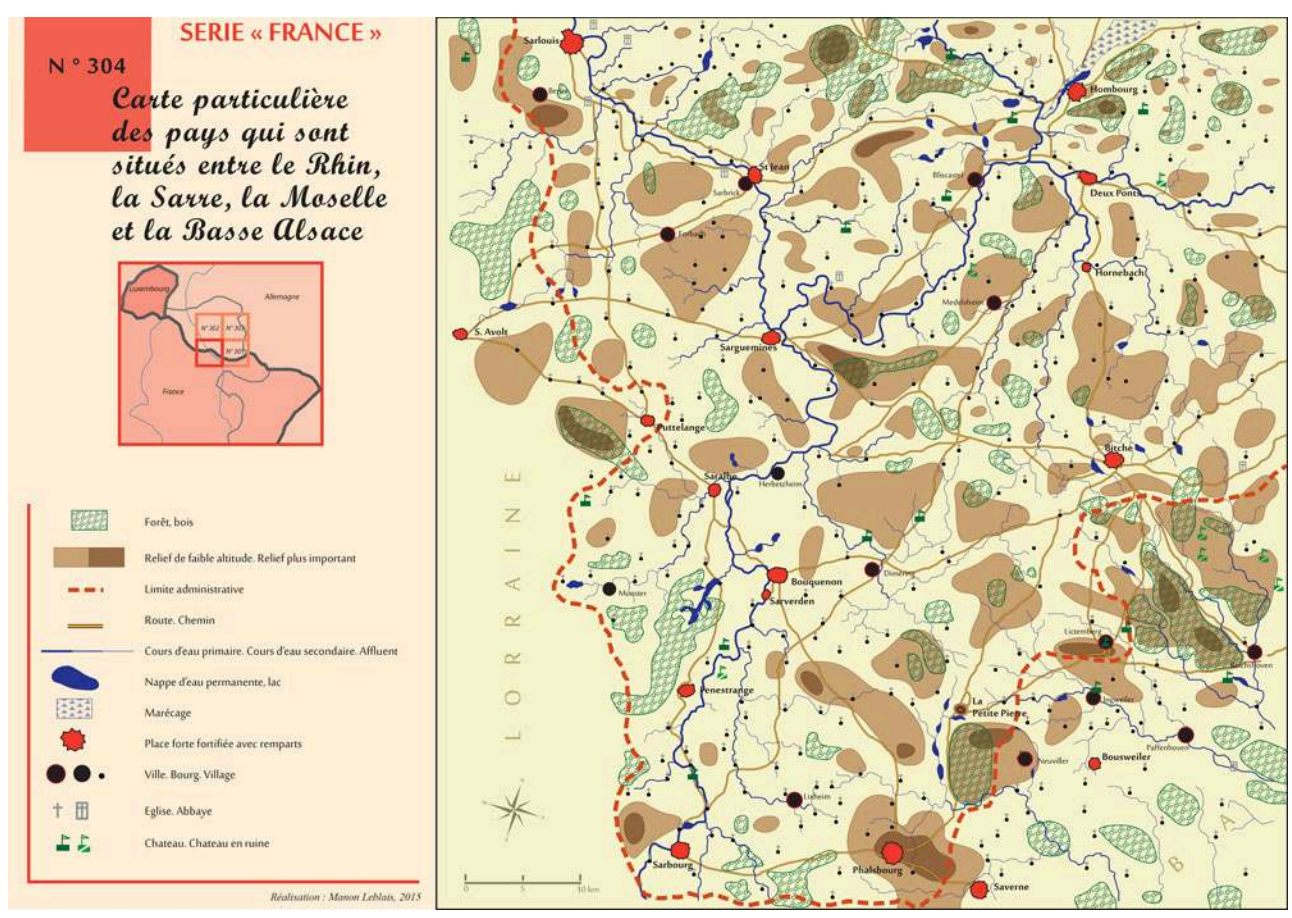

Projet Re-Cartes, exposition Cartologies, Strasbourg, 13-24 mars 2016.

Auteur : Manon Leblois. Master Carthagéo, janvier 2016 
11 L'inspiration de la carte «à plier» était également présente chez les étudiants toulousains du master SIGMA (http://sigma.univ-toulouse.fr/) avec le plan de Rome version IGN série bleue (figure 7) de Sébastien Peillet, répondant aux conventions de la carte IGN au 1:25000 et qui reprend l'emprise du plan de Rome édité autour des années 1710-1720 déjà cité ci-dessus. Insérés dans la maquette usuelle sur le plan graphique, le titre non conventionnel, la numérotation " $0000 \mathrm{RT}$ » et l'échelle de la carte (1:18000) sont les indices qui vont interpeller le lecteur. La lisibilité des toponymes est difficile (le détourage est à améliorer). Le format numérique ne rend pas justice à l'attractivité du tirage papier plié que quelques visiteurs de l'exposition ont escamoté discrètement.

Figure 7. La Rome du XVIII' siècle

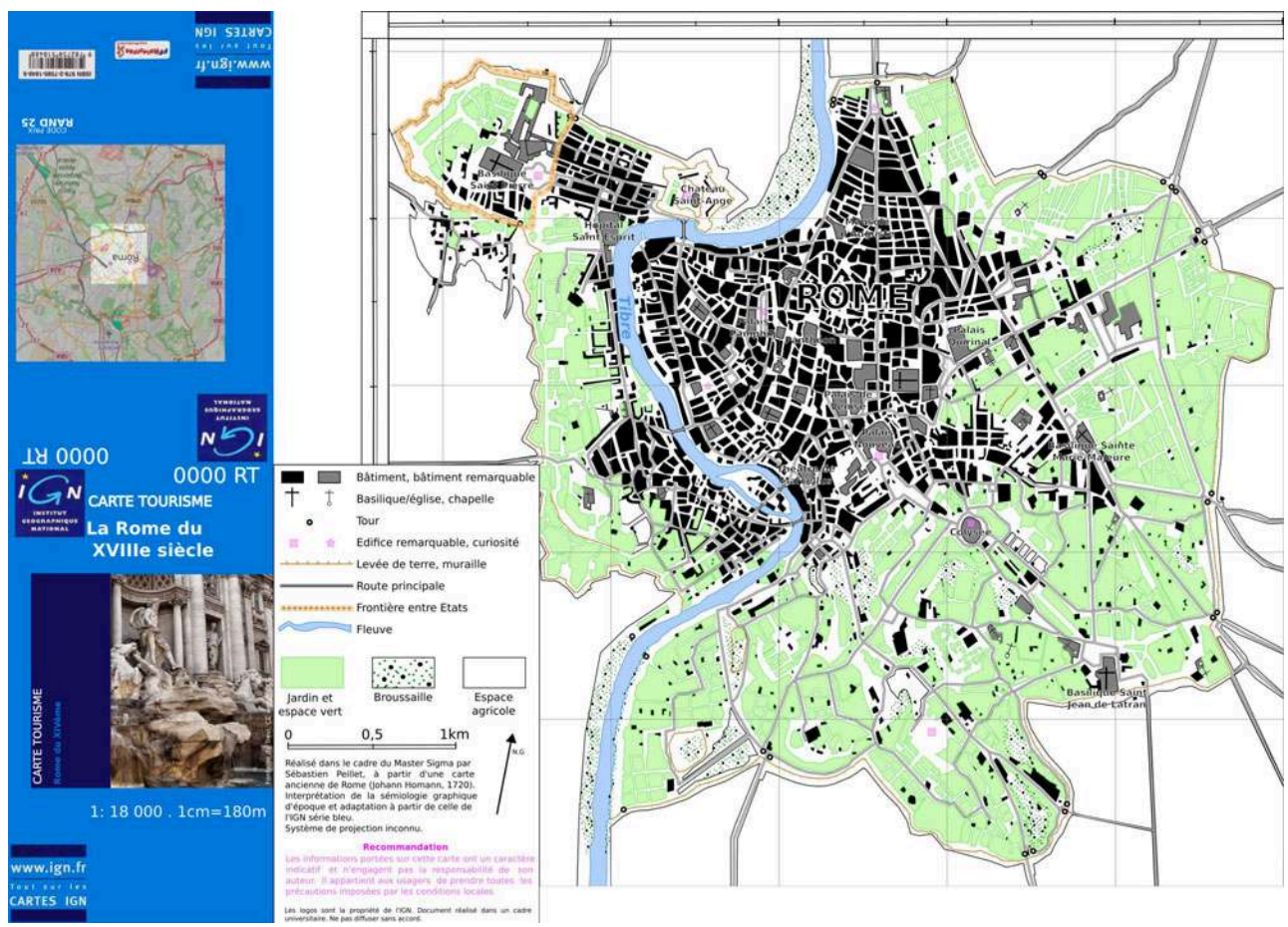

Projet Re-Cartes, exposition Cartologies, Strasbourg, 13-24 mars 2016

Auteur : Sébastien Peillet. Master SIGMA, janvier 2016

\section{Double jeu sur les codes anciens et contemporains}

Dix-huit étudiants de la promotion 2015-2016 du Master SIGMA de Toulouse ont créé un jeu de 13 cartes.

Laurent Jégou a repris l'idée initiale et a proposé aux étudiants, qui travaillaient seuls ou en binôme, de choisir entre deux modalités :

- jouer avec les codes d'une carte ancienne pour cartographier un territoire contemporain, ce qui a produit, par exemple, la carte Centre de Toulouse et ses différents quartiers de Nicolas Karasiak et Aenne Ramm ;

- redessiner une carte ancienne à l'aide de codes contemporains (y compris issus du champ de l'Art), avec comme résultat, par exemple, la Grèce antique façon OpenStreetMap (http:// www.openstreetmap.org/) de Raphaël Sorlin-Racine. 
Figure 8. Centre de Toulouse et ses différents quartiers

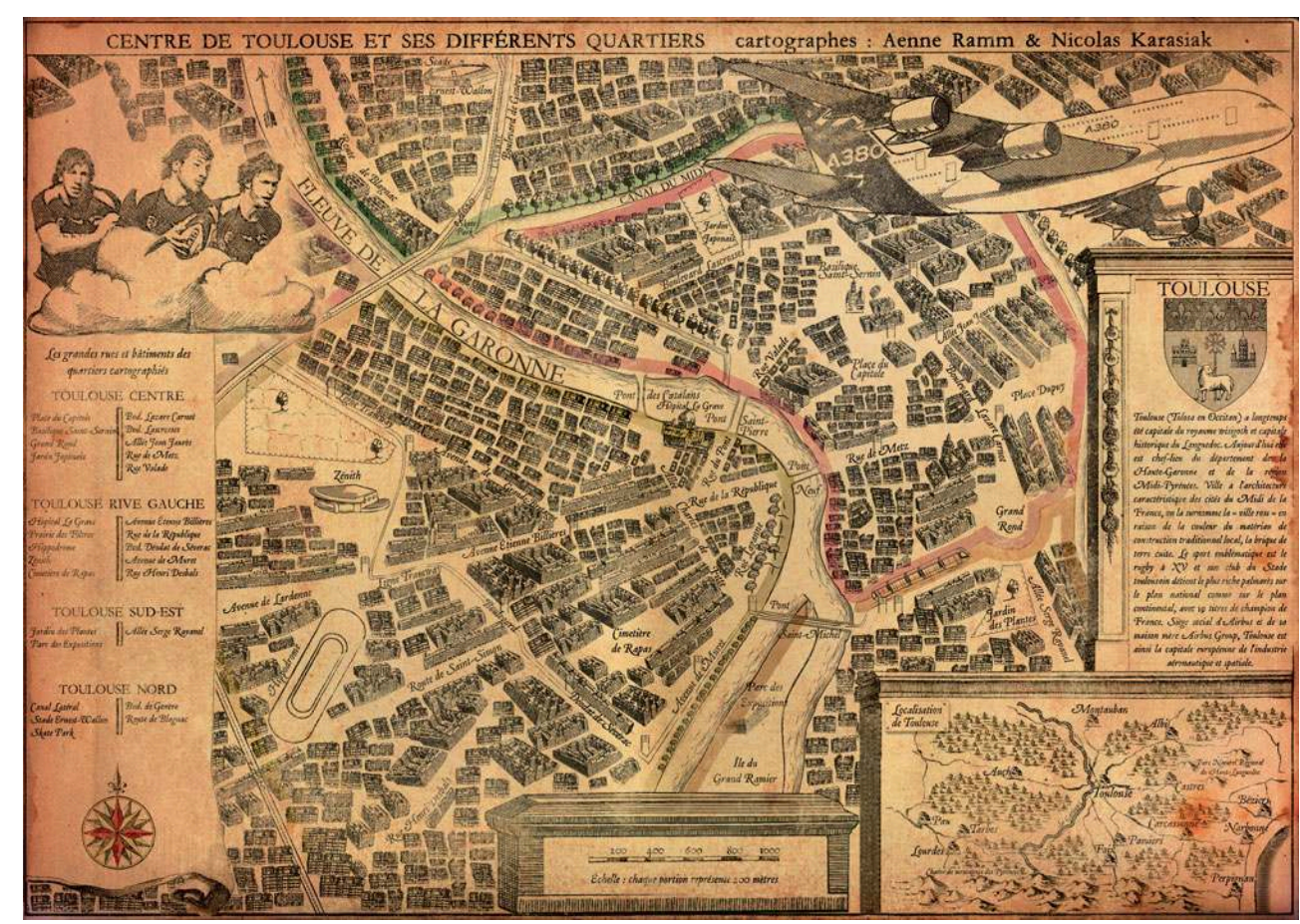

Projet Re-Cartes, exposition Cartologies, Strasbourg, 13-24 mars 2016.

Auteurs : Nicolas Karasiak et Aenne Ramm. Master SIGMA, janvier 2016

Figure 9. Græciæ antiquæ

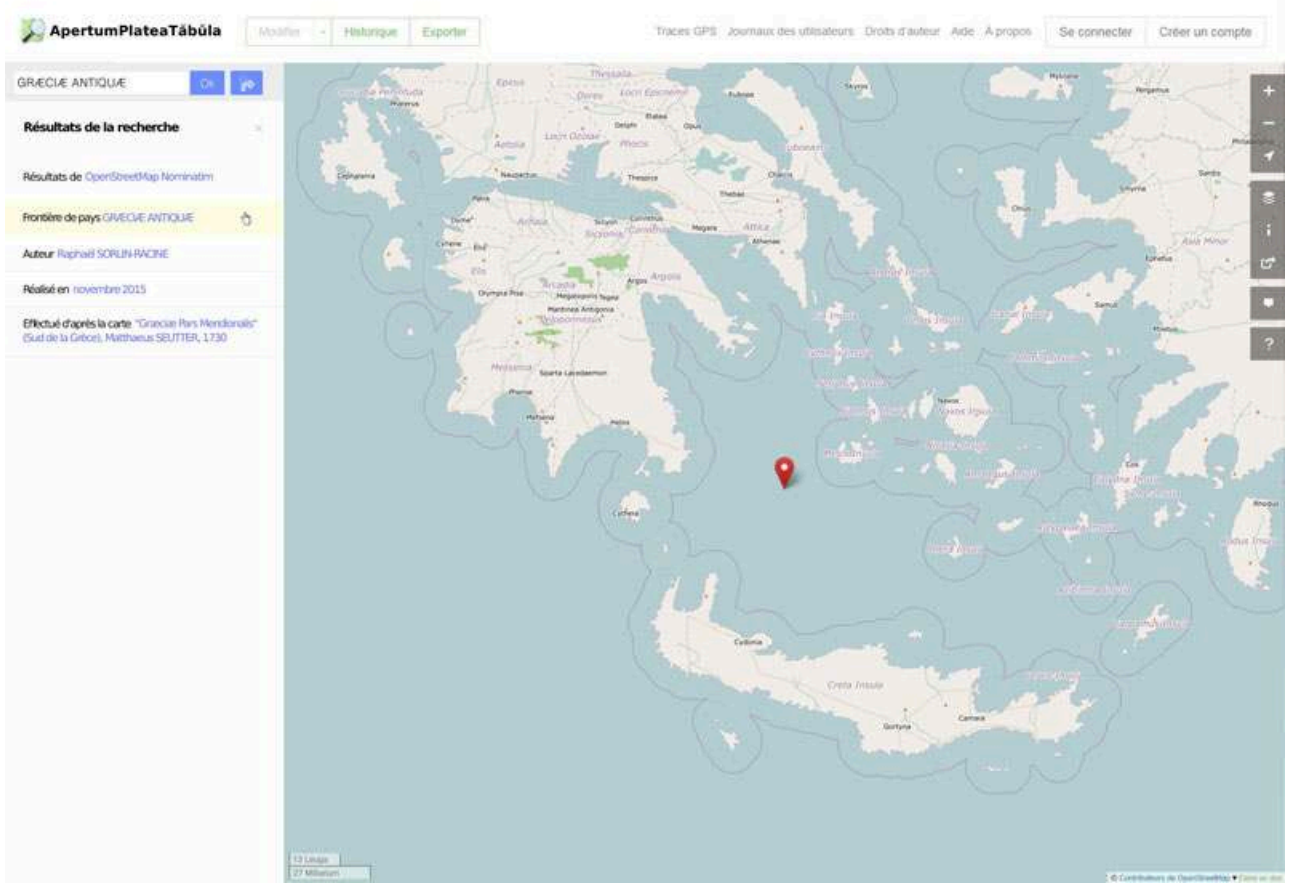

Projet Re-Cartes, exposition Cartologies, Strasbourg, 13-24 mars 2016.

Auteur : Raphaël Sorlin-Racine. Master SIGMA, janvier 2016 
La carte du Centre de Toulouse et ses différents quartiers (figure 8) est inspirée de deux documents sources déjà cités ci-devant, le plan de Rome Urbis Romce veteris ac Modernce Accurata Delineati pour la partie plan de la ville, et la carte sans titre de 1907 (Carte particulière des Pays qui sont situéz entre le Rhein, la Sarre, la Moselle et la Basse Alsace...) pour l'encart représentant la situation de Toulouse à plus petite échelle.

Sur cette représentation très riche, les étudiants ont composé et joué avec un grand nombre de codes graphiques et composants de la carte, allant jusqu'à la couleur du papier, la simulation de l'impact de sa manipulation et des conditions de stockage.

Pour souligner quelques éléments de la mise en scène cartographique, du choix et de l'organisation des composants de la carte, on retrouve le blason et l'histoire de la ville, le bloc de l'échelle ou la liste des territoires cartographiés, finement insérés sous la forme d'un parchemin ou de socles en pierre taillée autour du plan. Les anges qui du ciel observent le monde terrestre, que l'on retrouve sur nombre de cartographies anciennes, comme cette Mapa Geografico De America Meridional ( https:// www.davidrumsey.com/luna/servlet/detail/RUMSEY 8 1 240068 5512119:-Sheet-2-Mapa-Geografico-De-Americ?

sort=Pub_Date\%2CPub_List_No\%2CPub_List_No\%2CSeries_No\&qvq=q:Pub_List_No\%3D\%226931.000\%22;sort:Pub_Date\%2C de la cartothèque David Rumsey, les joueurs de l'équipe de rugby se penchent, avec bienveillance pour Alexis Palisson au centre, sur leur ville. Toujours dans la catégorie de ces éléments illustratifs et figuratifs propres à ces cartographies anciennes, au même titre que les monstres marins et navires des cartes marines ou les scènes de la vie des colonies des cartes d'Afrique dans les Atlas, l'Airbus A380 ancre et perpétue une image de la cité contemporaine.

7 Du côté des choix graphiques, que ce soit sur l'encart à petite échelle situé en bas à droite ou sur le plan, les auteurs copient les symboles très figuratifs et imitent la typographie des deux cartes sources, au sein desquels vont se fondre des lieux d'ancrage du présent, comme le zénith ou les stations de tramway.

La carte Grocice antiquae ( figure 9) sur la base de la carte source (http:// docnum.unistra.fr/cdm/compoundobject/collection/coll18/id/5/rec/1) du même nom (Auteur : Seutter, Matthäus. Date : v. 1734) est une simulation de page web issue d'une recherche sur le toponyme Grcecice antiqua sur l'interface cartographique du projet OSM (OpenStreetMap) ${ }^{2}$. L'auteur de la proposition cartographique compose sa mise en page en plaçant les différents menus de l'outil en ligne: modifier, historique, exporter, traces GPS, etc., ainsi que toutes les icônes appelant les fonctions de navigation ou d'information concernant la carte sur la gauche.

Un gros travail de sélection de l'information (tracé, symboles figuratifs et densité des toponymes) a été mené pour arriver à une carte dépouillée correspondant au design graphique de la Grèce à la façon d'OpenStreetMap (OSM).

Les zones montagneuses présentes sur la carte source disparaissent. Les contours terrestres, la délimitation des royaumes de l'antiquité (en tiretés mauve pâle) ainsi que les routes principales (dans une couleur orange-marron), les cours d'eau et les lacs sont redessinés. Des aplats de couleur verte reprennent l'emprise de petits arbres (zone forestière ou arboriculture ?) dans le Peloponnesus. Les plans d'eau apparaissent dans leurs contours d'origine. Afin d'imiter au mieux la représentation contemporaine, le cartographe a ajouté les tracés des limites des eaux territoriales sous forme d'enveloppes (zones tampons) autour des côtes. À une certaine échelle et pour les 
emprises où l'espace maritime est présent, ces traits épais dans les tons mauves et leur forme arrondie marquent l'identité visuelle de l'affichage cartographique de l'interface OSM.

21 L'échelle est calculée et exprimée en mesure d'époque (dont la valeur de conversion en kilomètres varie légèrement selon les sources), le mille romain (milliarum) et la lieue romaine (leuga). Elle s'accorde avec le titre et la description de la carte rédigée en latin dans le cartouche, texte qui aurait pu être repris dans la réécriture cartographique pour renseigner le menu, soit au niveau du résultat de la requête, soit pour simuler l'utilisation du système d'annotation de l'interface. Dans la même veine, on regrettera que l'auteur n'ait pas développé une légende derrière le (i) de la rubrique information.

Il a été difficile, lors de l'exposition, ou pour le texte présent, de sélectionner un nombre limité de cartes, car c'est la richesse des expressions qui caractérise l'intérêt et la dimension pédagogique du projet. Nous terminons par cette dernière proposition de Céline Durupt et Aurélien Schmitt qui se distingue par son esthétique et la maîtrise des couleurs. La Carte des côtes anciennes et bathymétrie moderne méditerranéennes s'appuient sur de nombreuses ressources en ligne: une carte ancienne, Seale Map or Chart of the Mediterranean Sea (https://commons.wikimedia.org/wiki/File : 1745_Seale_Map_or_Chart_of_the_Mediterranean_Sea_-_Geographicus__Mediterranean-seale-1845.jpg), pour définir l'emprise territoriale, l'image de l'Agence américaine d'observation océanique et atmosphérique (NOAA, National Oceanic and Atmospheric Administration) pour la bathymétrie et un grand nombre de références anciennes pour construire les différents cartouches et décors qui ancrent dans l'ancien temps la représentation.

Figure 10. Carte des côtes anciennes et bathymétrie moderne méditerranéennes

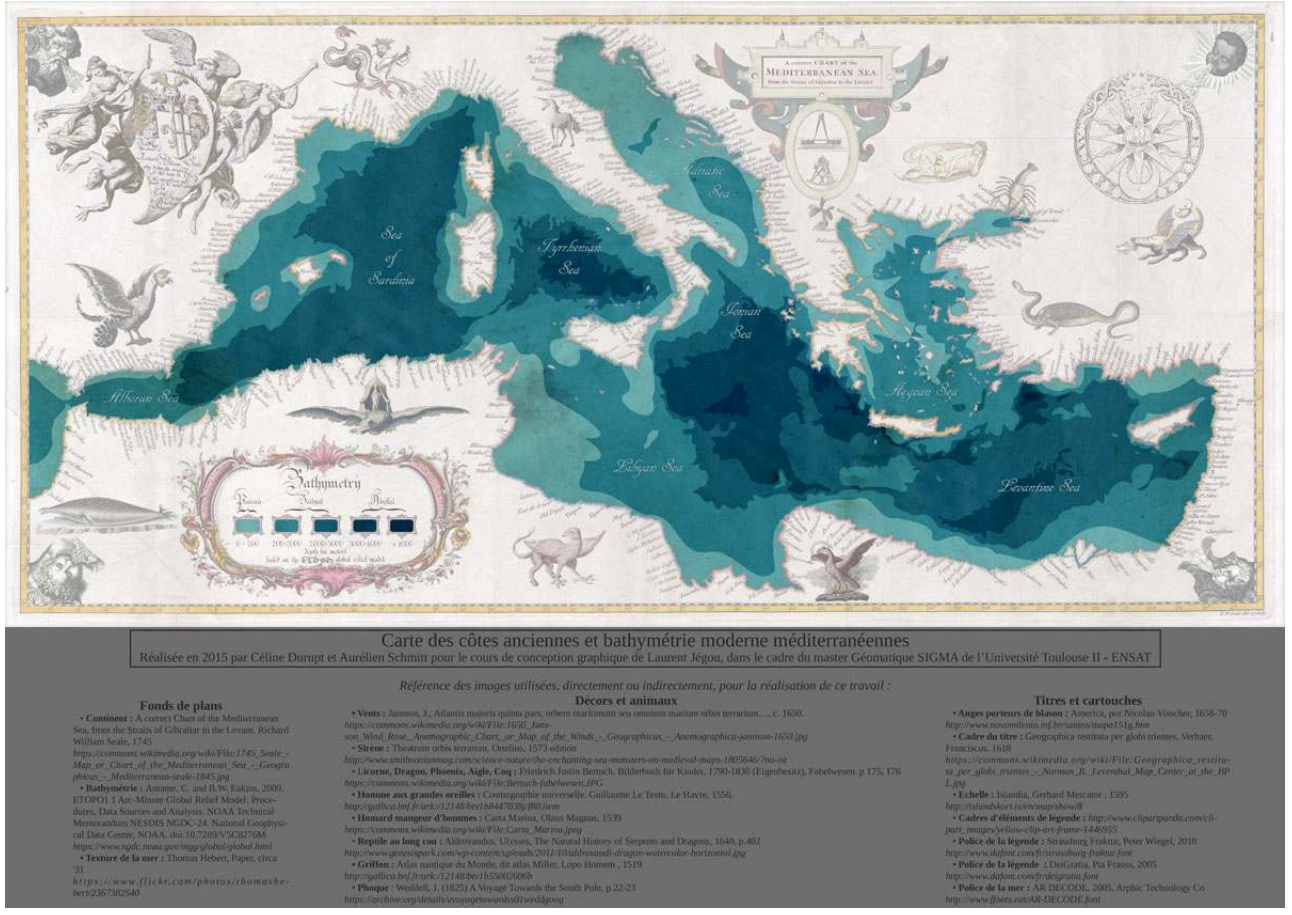

Projet Re-Cartes, exposition Cartologies, Strasbourg, 13-24 mars 2016

Auteurs : Céline Durupt, Aurélien Schmitt. Master SIGMA, janvier 2016 
Il n'existe malheureusement pas d'archive numérique des explorations cartographiques menées par les étudiants de l'InSituLAB sur la base de La sémiologie dans tous les sens. Seules subsistent quelques traces photographiques, comme Albutopies, une invitation au voyage dans les reliefs blancs du monde de Petronille Camphuis qui échantillonne les espaces des cartes et transcrit les symboles des villes et montagnes en volume (figure 11).

Figure 11. Albutopies

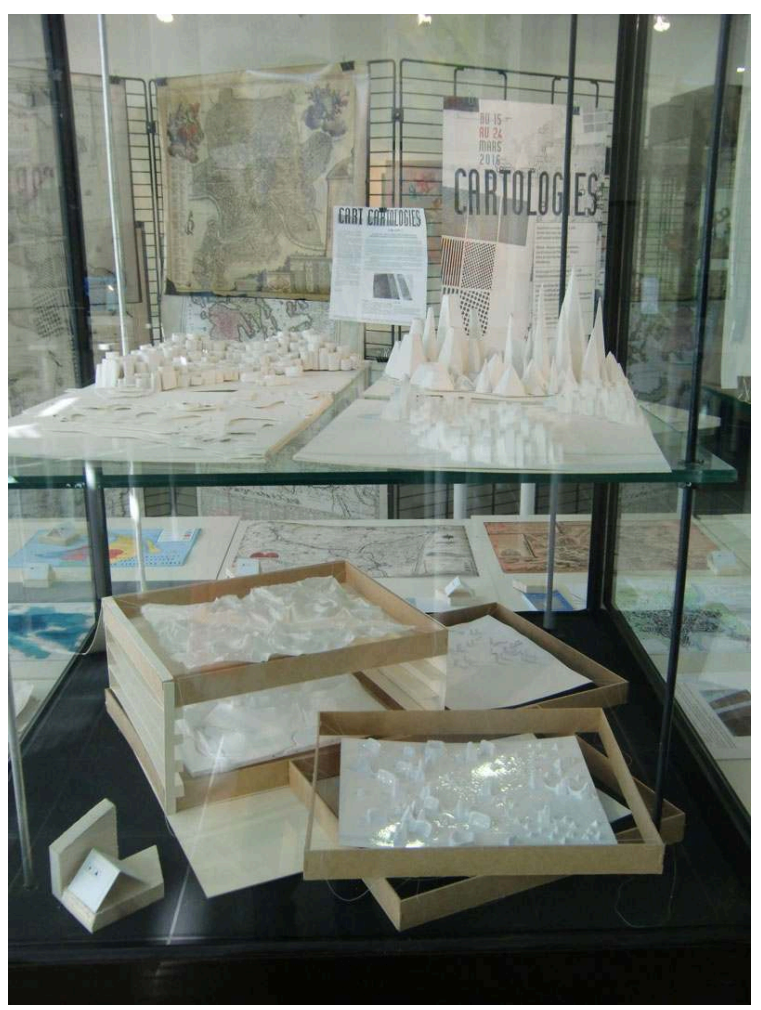

Projet Re-Cartes, exposition Cartologies, Strasbourg, 13-24 mars 2016

Auteur : Pétronille Camphuis. InSituLAB, janvier 2016. Photographie : A.-C. Bronner, 2016

\section{BIBLIOGRAPHIE}

BERTIN J. (1967). La Sémiologie graphique, diagrammes, réseaux, cartes. Paris : Éd. Gauthiers-Villars ; Paris/La Haye : Éd. Mouton et $C^{\mathrm{ie}}$; Paris : École Pratique des Hautes Études, 431 p.

\section{NOTES}

1. Colloque Temps Art \& Cartographie - La sémiologie dans tous les sens organisé les 16-18 mars 2016 à Strasbourg. L'exposition Cartologies est née de plusieurs mois de travail portés par Anne-Christine 
Bronner, cartographe au Laboratoire image, ville, environnement (CNRS-université de Strasbourg), Nicolas Couturier et Bruno Lavelle, enseignants et designers à l'InSituLAB, lycée Le Corbusier, Illkirch-Graffenstaden, et l'implication de leurs collègues pour accompagner et encadrer les différents travaux menés. Lieu : 14-23 mars 2016, MISHA (Maison Interuniversitaire des Sciences de l'Homme-Alsace), Université de Strasbourg.

2. La charte graphique du site OpenStreetMap, de la carte et ses composants, les fonctionnalités évoluent régulièrement. La carte proposée se base sur l'interface à l'époque de l'exercice (hiver 2015-2016).

INDEX

Mots-clés : cartographie expérimentale, sémiologie graphique, charte graphique, cartes anciennes

\section{AUTEURS}

\section{ANNE-CHRISTINE BRONNER}

Laboratoire Sage, CNRS-Université de Strasbourg

LAURENT JÉGOU

UMR LISST, CNRS-université Toulouse

\section{CHRISTINE ZANIN}

UMS RIATE, Géographie-cités, CNRS-Université Paris Diderot-Paris 7 